\title{
Le pouvoir des sentiments : Kluge, Adorno,
} Ferenczi

Die Macht der Gefühle: Kluge, Adorno, Ferenczi

The power of feelings: Kluge, Adorno, Ferenczi

Grégory Cormann et Jeremy Hamers

\section{(2) OpenEdition}

Journals

Édition électronique

URL : http://journals.openedition.org/ceg/1108

DOI : 10.4000/ceg. 1108

ISSN : 2605-8359

Éditeur

Presses Universitaires de Provence

Édition imprimée

Date de publication : 18 décembre 2015

Pagination : 69-88

ISBN : 979-1-03200-020-5

ISSN : 0751-4239

Référence électronique

Grégory Cormann et Jeremy Hamers, «Le pouvoir des sentiments : Kluge, Adorno, Ferenczi », Cahiers d'Études Germaniques [En ligne], 69 | 2015, mis en ligne le 17 décembre 2017, consulté le 25 novembre 2020. URL : http://journals.openedition.org/ceg/1108 ; DOI : https://doi.org/10.4000/ceg.1108

Tous droits réservés 


\section{Le pouvoir des sentiments : Kluge, Adorno, Ferenczi Grégory CORMANN, Jeremy HAMERS \\ Université de Liège}

\section{Faire un film après Adorno}

Dans sa célèbre conférence «Éduquer après Auschwitz ${ }^{1}$ », Adorno donne un diagnostic grave de la société de son époque. Selon le sociologue, cette société est frappée par une «froideur universelle» dont les symptômes sont l'indifférence à l'égard d'autrui, le refoulement de l'angoisse, l'anéantissement de toute « capacité de résistance » des sujets, le durcissement émotionnel et physique des êtres humains. Pour Adorno, cette froideur est un « trait anthropologique fondamental ${ }^{2}$ ». Quelques années plus tôt, dans ses Lebensläufe ${ }^{3}$, Alexander Kluge pose les prémisses littéraires d'une théorie des émotions qui se trouve aux antipodes de toute généralisation de la froideur: cette théorie accorde en effet à tout être humain une capacité inébranlable de produire des souhaits et de protester, c'est-à-dire de réagir par la production d'émotions, quelque grave que soit la situation à laquelle il est confronté. A priori, entre Adorno et Kluge, deux conceptions des émotions s'opposent, et ce, à deux niveaux au moins. Kluge imagine un sujet dont les émotions ne peuvent être totalement anéanties alors qu'Adorno donne des exemples d'individus devenus des êtres pétris de froideur « totale ». Alors qu'Adorno réfléchit à la possibilité d'éduquer l'être humain après que ce dernier a subi ou orchestré l'horreur (quitte à commencer par le petit enfant chez lequel se forme déjà ce qui aboutirait plus tard à l'horreur ${ }^{4}$ ), Kluge imagine un sujet toujours déjà dépositaire d'une capacité inaliénable de s'émouvoir, une capacité originaire de la petite enfance, qui lui permet de protester contre le malheur subi, sans devoir y être spécifiquement éduqué 5 .

Theodor W. Adorno, «Éduquer après Auschwitz », in Modèles critiques, trad. de 1'all. par M. Jimenez et É. Kaufholz, Paris, Gallimard, 2003, p. 235-251.

2 Ibid., p. 248.

3 Alexander Kluge, Lebensläufe, Stuttgart, Henry Coverts Verlag, 1962. Dernière édition: A. Kluge, Lebensläufe, Frankfurt am Main, Suhrkamp, 2003.

4 «Erziehung wäre sinnvoll überhaupt nur als eine zu kritischer Selbstreflexion. Da aber die Charaktere insgesamt, auch die, welche im späteren Leben die Untaten verübten, nach den Kenntnissen der Tiefenpsychologie schon in der frühen Kindheit sich bilden, so hat Erziehung, welche die Wiederholung verhindern will, auf die frühe Kindheit sich zu konzentrieren. » Theodor W. Adorno, « Erziehung nach Auschwitz », in Stichworte. Kritische Modelle 2, Frankfurt am Main, Suhrkamp, 1969, p. 87.

5 «Die menschlichen Äcker, auf denen Vertrauen wächst, können in ihrer Produktion nicht innehalten. Wir haben keine Möglichkeit, im Mißtrauen, in dauerhaftem Zweifel, in Verweigerung und 
La tension entre deux conceptions du sujet rapidement brossées ici devient particulièrement explicite lorsqu'on se penche sur la théorie du cinéma d'Alexander Kluge. Kluge n'a eu de cesse, depuis les années soixante-dix, de prôner un cinéma en mesure de susciter la production d'émotions chez chaque spectateur pris individuellement, ce qui n'est pas sans rappeler le projet éducatif adornien défendu dans «Éduquer après Auschwitz » lorsque le philosophe francfortois en appelle au travail de conscientisation par un intellectuel/réalisateur TV/éducateur ${ }^{6}$. Mais, on le sait, le cinéma politique de Kluge s'écarte aussi de ce projet éducatif en tant qu'il est adossé à une autonomie propre à chaque spectateur. Selon le réalisateur, le cinéma peut s'avérer politiquement efficace dans la mesure où il s'empare d'une capacité originaire de tout sujet à produire des émotions. L'opposition au projet d'éducation audiovisuelle d'Adorno est manifeste et permet de préciser la tension déjà dégagée en ouverture de ce texte: alors que le philosophe rêve d'un réalisateur capable de conscientiser les masses en vue d'un retour aux émotions, Kluge, en revanche, imagine un art grâce auquel tout spectateur est d'emblée, toujours déjà, un coauteur parce que toujours capable de produire des émotions. Pourtant, Kluge s'est souvent présenté comme un auteur « venant d'Adorno ${ }^{7} »$.

\section{La théorie des émotions d'Alexander Kluge}

Dans « Éduquer après Auschwitz », Adorno réagit à l'absence d'intérêt de la population allemande à l'occasion du Deuxième Procès d'Auschwitz qui s'est tenu à Francfort pendant près de deux ans, entre 1963 et 1965, dans une telle indifférence publique qu'il n'a pas été possible d'établir les faits dont étaient suspectés plusieurs dizaines d'exécutants du camp d'Auschwitz-Birkenau: confrontés à l'indifférence de l'opinion publique, les témoins allemands avaient en effet retiré leurs accusations ou déclaré qu'ils ne savaient plus, tandis que les accusés pouvaient revenir sur leurs aveux ou minimiser leur responsabilité. Pour Adorno, cette indifférence est synonyme d'une grande inquiétude: il en conclut que les conditions qui ont amené

\footnotetext{
Widerstand zu verharren (nicht einmal im Generalstreik). Menschen müssen das Vertrauen, das jedes junge Lebewesen mit auf die Welt bringt, verausgaben ». Alexander Kluge, avant-propos de Früchte des Vertrauens, Frankfurt am Main, Suhrkamp, 2009, p. 4 (pour une citation plus complète, voir plus bas). Cette confiance originelle s'exprime, selon Kluge, sous la forme de souhaits et d'émotions qui puisent leur « racine radicale dans le bonheur de l'enfant ». A. Kluge, «Wünsche und Fakten », in Klaus Eder, Alexander Kluge, Ulmer Dramaturgien. Reibungsverluste. Stichwort: Bestandsaufnahme, München, Carl Hanser, 1980, p. 94. À ce sujet voir aussi: Alexander Kluge, Die Kunst Unterschiede zu machen, Frankfurt am Main, Suhrkamp, 2003, p. 57.

6 Parce que le «système normal de l'école primaire qui pose souvent nombre de problèmes ne suffit pas à modifier cet état des choses », Adorno va en effet jusqu'à imaginer des émissions télévisées éducatives ainsi que des groupes d'éducateurs « qui se rendraient à la campagne ». Adorno, «Éduquer après Auschwitz », p. 241.

7 Au rapprochement effectué par son interviewer entre son œuvre et Héritage de ce temps d'Ernst Bloch, Kluge répond: «Je proviens davantage d'Adorno ». Alexander Kluge, Jochen Rack, «Erzählen ist die Darstellung von Differenzen », Neue Rundschau, ${ }^{\circ}$ 1, 2001. Cet entretien est reproduit en ligne sur le site de l'auteur : http:/www.kluge-alexander.de/zur-person/interviews-mit/details/artikel/erzaehlen-istdie-darstellung-von-differenzen.html.
} 
à la prise de pouvoir du nazisme se sont maintenant généralisées à une grande partie de la population qui fait montre d'une irresponsabilité totale à l'égard de son histoire récente.

Ce diagnostic s'inscrit dans la continuité de La Dialectique de la raison. Dans l'introduction de leur livre, Horkheimer et Adorno faisaient le constat désabusé que la conscience de leur époque n'autorisait à en produire qu'une analyse fragmentaire, soulignée par le sous-titre de l'ouvrage, Fragments philosophiques:

\begin{abstract}
Was wir uns vorgesetzt hatten, war tatsächlich nicht weniger als die Erkenntnis, warum die Menschheit, anstatt in einen wahrhaft menschlichen Zustand einzutreten, in eine neue Art von Barbarei versinkt. Wir unterschätzten die Schwierigkeiten der Darstellung, weil wir zu sehr noch dem gegenwärtigen Bewußtsein vertrauten. ${ }^{8}$
\end{abstract}

Horkheimer et Adorno ajoutent: «Les fragments que nous avons réunis ici prouvent cependant que nous avons dû renoncer à la confiance qui présida à nos débuts $^{9}$. \ Au cours de la rédaction de leur livre, la montée du nazisme et la Seconde Guerre mondiale les ont amenés à prendre de plus en plus en compte le caractère destructeur du progrès de la civilisation. Horkheimer et Adorno en sont ainsi venus à compliquer - jusqu'à sembler la renverser en son contraire - l'étude de la civilisation des mœurs depuis la Renaissance que Norbert Elias avait menée dans les années 1930 sur des bases théoriques proches ${ }^{10}$. Pour Horkheimer et Adorno, on pourrait résumer les choses en disant que «l'Europe a deux histoires »: d'une part, une histoire officielle et honorable - l'histoire de la civilisation des mœurs, qui étudie la façon dont les mœurs se pacifient à partir de la Renaissance dans les entourages royaux, avant de se propager dans le reste de la population; d'autre part, une histoire souterraine, dont on ne parle pas, qui est « constituée par le destin des instincts et des passions humaines refoulées, dénaturées par la civilisation ${ }^{11}$. » Mais, en réalité, on le comprend, il n'y a qu'une histoire, l'histoire de la dialectique de la raison, celle d'un renversement de la raison en son contraire: celle qui expose comment, à force de rationalisation et de civilisation des mœurs, la raison est devenue folle et a fini par éradiquer les émotions de l'homme.

8 Max Horkheimer, Theodor W. Adorno, Dialektik der Aufklärung. Philosophische Fragmente, Frankfurt am Main, Fischer Verlag, 1969 [1944], p. 1.

9 Max Horkheimer, Theodor W. Adorno, La Dialectique de la raison. Fragments philosophiques, trad. de l'all. par É. Kaufholz, Paris, Gallimard, 2011, p. 13.

${ }^{10}$ Norbert Elias, La Civilisation des mœurs, trad. de l'all. par P. Kamnitzer, Paris, Pocket, 2002 [1939]; La Dynamique de l'Occident, trad. de l'all. par P. Kamnitzer, Paris, Pocket, 2003 [1939]. Elias lui-même n'était certes ni à l'abri des dangers de son temps ni aveugle à leur égard, comme le remarquent par exemple Freddy Raphaël et Geneviève Herberich-Marx, «Elias ethnologue de 1'Allemagne contemporaine ", in Sophie Chevalier, Jean-Marie Privat (dir.), Norbert Elias. Vers une science de l'homme, Paris, CNRS Éditions, 2013, p. 227-239. On peut toutefois se demander, objectivement, si les processus de décivilisation identifiés par Elias sont bien, comme chez Horkheimer et Adorno, l'envers dialectique du progrès de la raison. On peut aussi remarquer, subjectivement, qu'Elias fonde la rédaction de ses ouvrages sur la «persévérance» en lui d'une confiance qui remonte à son enfance: «J'ai une intuition qui me donne l'assurance que tout ira bien, finalement, et j'attribue cela à l'énorme sentiment de sécurité dont j'ai joui en tant qu'enfant unique, grâce à l'amour de mes parents. » (Norbert Elias, «Interview biographique » avec A. J. Heerma van Voss et A. van Stolk (1984), in Norbert Elias par lui-même, Paris, Fayard/Pluriel, 2013, p. 24).

11 Horkheimer, Adorno, La Dialectique de la raison, p. 250. 
Aussi le rapport de l'homme au monde - son rapport aux autres hommes comme aux objets - est-il un rapport intégralement réifié, c'est-à-dire le rapport d'une chose à une autre ou à d'autres choses qu'on peut utiliser ou détruire à sa guise. Comme l'écrit Adorno dans Minima Moralia, « les gens ont désappris à donner ${ }^{12}$ »: le don semble avoir perdu son sens dans une société où le superflu va de pair avec un repli sur l'intériorité. Et les choses elles-mêmes ne sont plus, si on peut dire, des choses émotionnelles, qui reliaient jusque-là les hommes au monde et permettaient, grâce à leur " chaleur ${ }^{13}$ ", le développement des relations de générosité et de confiance. Selon Adorno, la discipline éducative allemande a franchi la limite: il ne s'agit plus d'une éducation du sang-froid ${ }^{14}$, il s'agit d'une imposition sociale totale qui a rendu les êtres humains insensibles et aveugles à la destinée de leurs prochains.

On comprend, dès lors, que ce qui intéresse Adorno dans «Éduquer après Auschwitz », c'est le processus historique de civilisation des mœurs qui diffuse jusque dans les couches sociales les plus basses les conditions de possibilité d'une froideur sociale toute civilisée qui, non seulement n'inhibe pas la violence, mais en libère les formes les plus brutales. Ainsi, lorsqu'il voit Anita G. en 1967, Adorno s'enthousiasme-t-il de reconnaître dans le premier long-métrage de son élève et ami, notamment dans la scène du tribunal au début du film - lorsque l'accusée Anita Grün, jugée pour le vol d'un pullover en plein été, déclare «en été aussi j'ai froid »-, une parfaite restitution de cette indifférence généralisée de la société allemande ${ }^{15}$.

Cette reconnaissance d'Adorno repose pourtant sur un malentendu. Kluge prend, dirait-on, le contrepied de son maître: là où Adorno insiste sur l'insensibilité des rapports humains réifiés, Kluge a commencé d'élaborer une théorie des émotions, une théorie des sentiments qui est certainement une des marques de fabrique de l'ensemble de son œuvre. Ainsi, alors qu'Adorno voit, dans Auschwitz, le surgissement brutal de l'histoire souterraine des passions refoulées par la civilisation, Kluge, dans sa monumentale Chronique des sentiments ${ }^{16}$, fait inlassablement le récit de l'impossible refoulement de sentiments qui ne cessent, dans les situations les plus difficiles, de manifester une protestation de l'être humain, de produire une différence dans le monde, si minime soit-elle. Pour Kluge, le projet d'un contrôle total des émotions est par conséquent tout simplement un projet impossible ${ }^{17}$.

${ }^{12}$ Theodor W. Adorno, Minima Moralia. Réflexions sur la vie mutilée, trad. de l'all. par É. Kaufholz et J.-R. Ladmiral, Paris, Payot, 2003 [1951], p. 52.

13 Ibid., p. 53.

${ }^{14}$ Selon la formule que Marcel Mauss applique aux techniques du corps dans son article pionnier: Marcel Mauss, «Les techniques du corps » [1936], in Sociologie et Anthropologie, Paris, PUF, 2004 [1950], p. 385. Nous verrons plus loin que la sociologie de Mauss est une des références et une des cibles théoriques d'Adorno tout au long de son œuvre.

15 Conformément aux courriers d'Adorno à Kluge des 13 mars et 26 mai 1967, reproduits en avant-propos du recueil de récits courts Stroh im Eis (livret accompagnant le film Landschaften mit Eis und Schnee). Cf. Alexander Kluge, Wer sich traut, reißt die Kälte vom Pferd, Berlin, Filmedition Suhrkamp, 2010, p. 4-5.

${ }^{16}$ Alexander Kluge, Chronik der Gefühle, Frankfurt am Main, Suhrkamp, 2000.

${ }^{17}$ On retrouve cette position fondamentale dans le film Die Macht der Gefühle, lorsque la voix off dit que « le contraire des sentiments ce sont les choses ». Ce qui signifie, si on retourne la formule, qu'il n'y a qu'une chose qui peut échapper à la réification, ce sont les sentiments. 
Si l'on devait résumer le projet de Kluge dans Chronique des sentiments (texte partiellement traduit en français en $2003^{18}$ ), on pourrait avancer que l'ouvrage fait alterner les passages qui mettent en scène l'effondrement de notre capacité de décision, ce que Kluge appelle « la paralysie au moment décisif ${ }^{19}$ ", et des récits qui manifestent la résistance, parfois ultime, à un tel refroidissement de notre volonté. Dans son discours pour la remise du Prix Kleist en 1985, Kluge soutenait déjà que la tâche de l'écrivain est de se faire le "gardien de la différence » des temps, à savoir de la distinction du passé, du présent et du futur - ce qu'il appelle la « grammaire des temps ${ }^{20}$. La possibilité de continuer à faire cette différence n'est rien d'autre pour Kluge que la possibilité même de prendre une décision: « Je ne peux rien ressentir sans éveiller en moi l'enfant que je fus et les parents qui ont influencé cet enfant. Je ne peux absolument pas parler sans espoir: je serais paralysé. Je ne peux pas me passer de ce moment où il semble qu'une décision peut encore être prise: nous appelons cela le présent ${ }^{21} »$. Aussi Kluge s'ingénie-t-il, dans sa Chronique, à décrire ce qu'il appelle des « réserves » de pensée et d'imagination, des réserves de temps ou de décision. La campagne de Russie de Napoléon en 1812, qui est comme une préfiguration de la défaite d'Hitler à Stalingrad, en est un bon exemple $^{22}$. Malgré l'évidence de la débâcle française, Kluge s'intéresse à la façon dont Napoléon utilise la " rigueur du froid» pour encore accomplir des actions à la hauteur de la Grande Armée. Ainsi, lorsqu'il décide d'interrompre sa retraite pour attaquer l'armée russe lancée à sa poursuite, il ne faut voir chez Napoléon, selon Kluge, aucune attitude suicidaire: Napoléon cherchait simplement « en se mettant dans des situations désespérées et insupportables [...], dans des situations extrêmes, [à] faire encore jaillir de lui un atome de riposte, de révolte intérieure ", autrement dit à « se maintenir psychiquement en mouvement ${ }^{23}$ ». Kluge ajoute: «Beaucoup ont dit qu'il cherchait la mort. Son ordonnance, qui le connaissait mieux, répondit: Il cherchait un reste de décision. Il était "gelé" 24 ». La propre expérience de Kluge, en 1945, sous les bombardements de Halberstadt, en est un autre exemple: malgré les bombardements, il est porté par la certitude d'être le seul rescapé et se demande simplement s'il aura sa leçon de piano le lendemain. Les exemples concernant ce « contre-capital » abondent dans l'œuvre de Kluge.

Die menschlichen Äcker, auf denen Vertrauen wächst, können in ihrer Produktion nicht innehalten. Wir haben keine Möglichkeit, im Mißtrauen, in dauerhaftem Zweifel, in Verweigerung und Widerstand zu verharren (nicht einmal im Generalstreik). Menschen müssen das Vertrauen, das jedes junge Lebewesen mit auf die Welt bringt, verausgaben. In dieser Hinsicht sind Menschen keine « rationalen », sondern « verschwenderische » Lebewesen. Das von Sigmund Freud so genannte « Ur-Vertrauen », ein Konto, von dem

18 Alexander Kluge, Chronique des sentiments, trad. de l'all. par P. Deshusses, Paris, Gallimard, 2003 [2000].

19 Ibid., p. 60.

${ }^{20}$ Alexander Kluge, «La différence » [1985], in De la grammaire du temps, trad. de 1'all. par A.-É. Delatte, Paris, L’Harmattan, 2003, p. 98.

${ }^{21}$ Ibid., p. 87.

${ }^{22}$ Ibid., p. 89.

23 Kluge, Chronique, p. 149, n. 2.

24 Ibid., p. 149-150. 
wir bis zum Tod täglich Abbuchungen vornehmen, ohne es zu erschöpfen, ist eine Mitgift der Evolution: Menschen wären auf dem langen Marsch in die Gegenwart ohne dieses seltsame Gegen-Kapital nicht übriggeblieben. ${ }^{25}$

Ce qui intéresse Kluge dans l'enfance, ce qui le fait se tourner vers les premières années de la vie humaine, c'est la croyance que nous avons alors que « le monde [est] bien intentionné à [notre] égard ».

In der Evolution haben jene Lebewesen überlebt, die in den ersten Jahren ihres Daseins glauben, daß die Welt es gut mit ihnen meint. Betrachtet man unser Jahrhundert, ist das sicher ein großer Irrtum. Die Welt meint es nicht gut mit den Toten von Verdun, sie meint es nicht gut mit den Holocaust-Toten. Insofern läßt sich ganz objektiv sagen: Dies ist Ideologie, ein Irrtum, ein wunderbarer Irrtum allerdings, der Kräfte gibt. Das würde auch Adorno unterschreiben. Darum heißt mein letztes Kapitel in der « Chronik der Gefühle » - « Der lange Marsch des Urvertrauens ». ${ }^{26}$

On identifie donc clairement l'écart de Kluge par rapport à Adorno: Kluge s'en remet à une « erreur » qui s'est répétée sans cesse dans l'histoire, à une illusion qui, bien que l'on connaisse toujours déjà la fin (l'opéra, ça finit mal, au cinquième acte, dit-i ${ }^{27}$ ) donne la force de recommencer encore une fois. L'acteur, qui devrait savoir qu'il va mourir inéluctablement, a, chaque soir, représentation après représentation, les yeux plein d'espoir à l'acte I ${ }^{28}$. Plus précisément, pour échapper au destin funeste de la réalisation de la raison en son contraire dans l'histoire ${ }^{29}$, Kluge s'en remet à quelque chose qui, dans sa répétition fatale, échappe à l'histoire, à quelque chose qui est le non-historique absolu.

\section{Kluge, Ferenczi et le froid : le principe cinéma}

Comme la citation de l'avant-propos de Früchte des Vertrauens le suggère, c'est du côté de la psychanalyse qu'il faut se tourner. Kluge déclare lui emprunter le concept de « confiance originelle » (Urvertrauen). Ce faisant, on comprend le geste

${ }^{25}$ Kluge, avant-propos de Früchte des Vertrauens, p. 4. Comme nous allons le voir, le concept de « confiance originelle » est popularisé dans les années 1950 par certains psychanalystes comme Erik Erikson. Il n'est pas aisé d'identifier le concept chez Freud. La formule, qu'on peut aussi traduire par «confiance de base », renvoie peut-être à ce que Freud appelle "l'expérience de satisfaction » (Befriedigungserlebnis) qui est décrite dans l'Esquisse de psychologie scientifique de 1895. Voir sur ce dernier point: Jean Laplanche, Jean-Bertrand Pontalis, Vocabulaire de la psychanalyse, Paris, PUF, 2007 [1967], p. 150-151.

${ }^{26}$ Kluge, Rack, « Erzählen ».

27 « $[\mathrm{I}] \mathrm{n}$ jeder Oper, die von Erlösung handelt, wird im fünften Akt eine Frau geopfert», in Alexander Kluge, Die Macht der Gefühle, Frankfurt am Main, Zweitausendeins, 1984, p. 174.

${ }^{28}$ Nous faisons référence ici à l'interview d'un chanteur d'opéra dans le film Die Macht der Gefühle (1983), que Kluge interroge sur sa capacité à espérer toujours et encore à chaque fois qu'il monte sur scène.

29 Dans La Dialectique de la raison, Horkheimer et Adorno avaient aussi pour objectif de lutter contre la cécité croissante de la raison. L'autoréflexion de la raison doit à la fois soutenir que la raison est la condition de possibilité de l'Aufklärung et qu'elle porte déjà toujours en elle-même le "germe » de sa régression. L'objet privilégié de la réflexion doit être, par conséquent, la possibilité même de sa régression. 
que Kluge met en œuvre: jouer un autre Freud contre le Freud de Malaise dans la civilisation sur lequel, comme on l'a vu, Adorno faisait fond dans sa critique de l'éducation moderne. Selon Adorno, un grand mérite de Freud est d'avoir identifié dans le refoulement du corps - et de la sensibilité dans sa dimension la plus brute une des sources de ce qu'il appelle « le malaise dans la civilisation ». À l'inverse, Kluge va chercher du côté de la psychanalyse un besoin fondamental de confiance qu'éprouve l'enfant dans les tout premiers mois de sa vie. Aussi, alors qu'Adorno voulait expliquer l'absence d'angoisse et de responsabilité, Kluge s'intéresse, lui, à la confiance que l'enfant suffisamment bien accueilli par son entourage reçoit au moment de sa naissance, et qui lui permet de ne pas devoir immédiatement affronter les menaces existentielles que recèle le monde où il apparait.

Le concept de "confiance de base », Urvertrauen, en anglais basic trust, a été forgé par le psychanalyste Erik Erikson ${ }^{30}$ au début des années 1950 . On peut penser que c'est à lui que Kluge l'emprunte ${ }^{31}$. Mais, en plus profonde analyse, c'est à un autre psychanalyste, Sandor Ferenczi (1873-1933), un des élèves les plus proches de Freud, que Kluge fait certainement référence. Erikson se contente en effet de décrire les différentes phases critiques que connaît un enfant au cours de son développement, alors que Kluge, dans ses films et dans ses récits, ainsi que dans ses nombreux entretiens, insiste sur une histoire catastrophique de l'espèce humaine et de ses ancêtres. Ferenczi, lui, radicalise de façon prodigieuse la thèse courante à son époque que l'ontogenèse répète la phylogenèse. Nos ancêtres auraient ainsi surmonté de nombreuses catastrophes, et chacune de nos existences en constitue à la fois la répétition et la répétition du défi relevé par l'homme face à cette réalité difficile. Parmi ces catastrophes, Kluge semble s'accorder avec Ferenczi pour donner une place particulière à la période glaciaire (à la dernière grande période glaciaire) ${ }^{32}$.

Dans son article fondamental de 1913 sur « Le développement du sens de réalité et ses stades ", Ferenczi interroge d'abord en profondeur la définition de l'animisme enfantin que donnera Freud dans Totem et Tabou, à savoir la croyance de l'enfant dans la toute-puissance de sa pensée. On pourrait croire que Ferenczi ne fait que reprendre, sur le vif, pour la développer et en préciser la portée, la conception freudienne de la « mégalomanie ${ }^{33}$ » de l'enfant. Dans cette perspective, Ferenczi ne ferait que décrire les différents stades d'acquisition du principe de réalité au détriment du principe de plaisir. En réalité, il en va autrement. L'article de Ferenczi constitue une critique radicale, quoique proche d'elle, de l'anthropologie freudienne ${ }^{34}$. Si on

${ }^{30}$ Erik Erikson (1902-1993) : né à Francfort, il s'est formé à la psychanalyse à Vienne, auprès d'Anna Freud notamment, avant d'émigrer aux États-Unis en 1933. Il y élabore une théorie du développement de l'identité, notamment dans Childhood and Society (1950).

${ }^{31}$ Comme le fait Christopher Pavsek dans The Utopia of Film. Cinema and Its Futures in Godard, Kluge and Tahimik, New York, Columbia University Press, 2013.

${ }^{32}$ Voir notamment: Alexander Kluge, « Die Utopie Film », in Eder, Kluge, Ulmer Dramaturgien, p. 61 .

33 Sandor Ferenczi, «Le développement du sens de réalité et ses stades » [1913], in L'enfant dans l'adulte, Paris, Payot, 2006, p. 49.

34 Ferenczi écrit qu'il n'a pas pu tenir compte de Totem et Tabou lorsqu'il rédigeait son article. Mais sa proximité avec Freud ne permet pas de douter qu'il était depuis longtemps au courant du travail de son maître. Voir ibid., p. 45, n. 1. 
lit correctement Ferenczi, on se rend compte que l'enfant, après sa naissance, ne vit à aucun moment intégralement sous le principe de plaisir. Le « sens de réalité » est contemporain de la naissance, dût-il se développer selon différents stades: ce sont les réponses de l'entourage humain de l'enfant qui permettent à celui-ci d'accorder foi à ses croyances. De telles croyances constituent du coup, selon Ferenczi, autant de tentatives pour conjurer, de façon hallucinatoire, la toute-puissance originelle ${ }^{35}$.

Dans la seconde partie de l'article, Ferenczi élargit le spectre de son investigation sur le développement du sens de réalité à des considérations phylogénétiques. Selon lui, la perte de la toute-puissance que constitue pour l'enfant sa naissance rejoue l'ensemble des catastrophes géologiques et climatiques vécues par «les ancêtres de l'espèce humaine ", jusqu'à la dernière période glaciaire qui est "survenue à une époque où il y avait déjà certainement des êtres humains sur la terre ${ }^{36} »$. Cette ouverture phylogénétique est approfondie dans Thalassa, qui est publié en 1924 mais dont Ferenczi a jeté les bases lors de son service militaire, à partir de l'automne $1914^{37}$. Il y précise la façon dont il faut concevoir les réactions symboliques face aux « situations difficiles ${ }^{38}$ » que les individus rencontrent. Prenant de nouveau pour modèle le traumatisme de la naissance, il soutient avec force qu'il s'agit dans chaque cas de «jouer avec le danger ${ }^{39} »$ : la phase cannibalistique de l'enfant, l'acte sexuel ou encore le sommeil ou la conversion hystérique sont autant de façons de revivre « le plaisir de l'existence intra-utérine, l'angoisse de la naissance et enfin la joie renouvelée d'échapper heureusement à ce danger ${ }^{40} »$.

C'est ainsi que la thématique du froid, dont nous sommes partis, subit une inversion fondamentale : là où Adorno définissait notre condition actuelle par le froid, là où Adorno définissait le froid comme un point d'aboutissement de l'histoire des sociétés humaines, Kluge - à la suite des premiers textes de Ferenczi-place le froid au départ: pour lui, «nous provenons tous du froid», et la période glaciaire nous a appris à « avoir des impressions ${ }^{41} »$. Aussi, là où il signifie pour Adorno insensibilité et indifférence, le froid est-il chez Kluge l'expression d'une capacité élémentaire

35 À tout prendre, c'est lorsque l'homme dort qu'il reproduit adéquatement la situation intra-utérine en se détournant des tâches à accomplir (assouvissement de ses besoins, réactions aux sollicitations et contraintes externes) de façon à pouvoir assurer sa croissance ou la régénération de ses forces.

36 Ibid., p. 67.

${ }^{37}$ Sandor Ferenczi, Thalassa. Psychanalyse des origines de la vie sexuelle, Paris, Payot, 2002 [1924], « Introduction », p. 47.

${ }^{38}$ Ibid., p. 70, 104 et 108.

39 Ibid., p. 107. Ferenczi se place ici dans la continuité des descriptions freudiennes du jeu de la bobine dans Au-delà du principe de plaisir.

40 Ibid., p. 118. Dans Thalassa, Ferenczi modifie certes le cadre de sa spéculation phylogénétique. Il fait encore allusion aux difficultés de la dernière période glaciaire (p. 148), mais c'est alors « l'assèchement des océans » (p. 113) qui est présenté comme le danger véritable, la vraie catastrophe qui a marqué l'histoire de l'humanité. Si l'existence individuelle répète l'histoire de l'espèce, c'est, selon le grand livre de 1924, que la naissance rejoue la nécessité qu'a jadis représentée pour de nombreux animaux la sortie de la mer de terres jusque-là immergées. On peut penser que Ferenczi se rallie là, finalement, aux analyses symboliques de l'eau que Freud avait proposées entre-temps dans son Introduction à la psychanalyse. Cf. les pages que Freud consacre au symbolisme de l'eau dans les rêves, in Sigmund Freud, Introduction à la psychanalyse, Paris, Payot, 1973 [1917], p. 145.

${ }^{41}$ Kluge, Chronique, p. 42. 
de différenciation et de défi envers la réalité telle qu'elle est. Le point n'est pas anecdotique, dans la mesure où la même inversion chronologique vaut aussi, selon Kluge, pour le cinéma lui-même. Pour Kluge, en effet, le cinéma n'est pas qu'une invention artistique récente (du coup unilatéralement passible de la critique que l'on peut faire des productions culturelles de masse). Comme il n'hésite pas à le répéter, le cinéma ne peut pas non plus être réduit à une certaine invention technique. Le cinéma est, dans son principe, consubstantiel au travail de l'esprit humain, à cette capacité de mouvement intérieur qu'est l'esprit humain. Un tel « cinéma » existe depuis plusieurs millénaires. Ainsi que le précise un passage essentiel de Kluge sur le «Principe cinéma »: «Depuis la période glaciaire à peu près (ou plus tôt), sont en mouvement dans la tête de l'homme - en partie pour des raisons anti-réalistes, à savoir comme protestation contre l'insupportable réalité - des flux d'images, ce qu'on appelle des associations ${ }^{42}$ ». Dans cette note de 1979, Kluge met explicitement le cinéma, comme art " obscur et sans grade », à l'écart des " chemins tortueux du malaise dans la culture (Sigmund Freud) »: le cinéma est une expérience millénaire qui se fonde sur un " matériau brut » qui ne cesse de traverser l'esprit des hommes depuis des millénaires (rires, souvenirs, idées), et qui de cette façon rapproche ceuxci sans avoir à en passer par ce que Kluge appelle « la simple éducation ${ }^{43}$ ».

\section{La théorie du cinéma d'Alexander Kluge}

L'intérêt particulier d'Alexander Kluge pour le cinéma repose sur le fait qu'il « fonctionne comme l'esprit humain » du sujet protestataire: par sauts successifs, déplacements et mises en rapport d'éléments hétérogènes, ce qui lui permet d'avancer que «même lorsque les projecteurs se seront tus, il y aura toujours [...] quelque chose qui fonctionnera comme le cinéma ${ }^{44} »$. Selon l'auteur, le cinéma produit en effet d'innombrables liens entre des images qui se trouvent à l'écran et les images dont chaque être humain est dépositaire en tant qu'héritier de toutes les générations qui l'ont précédé. La rencontre de deux images sur l'écran peut, selon Kluge, produire de multiples troisièmes images dans la tête du spectateur, ce qui transforme, selon la formule consacrée par l'auteur allemand, tout spectateur en un auteur. L'efficacité politique de ce « cinéma des associations », comme l'appelle Kluge, réside dans le fait que le spectateur peut s'affranchir de la fatalité que semble lui imposer la seule représentation (réaliste) du monde en protestant, c'est-à-dire en produisant des émotions, en se dressant contre toute situation difficile par la production d'autres images ${ }^{45}$.

${ }^{42}$ Alexander Kluge, «L'utopie cinéma » (1979), in L'Utopie des sentiments. Essais et histoires de cinéma, éd. par Dario Marchiori, trad. de l'all. par C. Jouanlanne et V. Pauval, Lyon, Presses universitaires de Lyon, 2014, p. 121.

${ }^{43}$ Kluge insiste sur la « proximité particulière [du cinéma] au spectateur et à l'expérience » (ibid.).

44 «Auch wenn die Kinoprojektoren einmal nicht mehr rattern, wird es, das glaube ich fest, etwas geben, das wie Kino funktioniert», in Alexander Kluge, avant-propos de Geschichten vom Kino, Frankfurt am Main, Suhrkamp, 2007, p. 7.

45 Alexander Kluge, « Ein Hauptansatz des Ulmer Instituts », in Eder, Kluge, Ulmer Dramaturgien, 
Ce montage qui met en quelque sorte le spectateur au travail puisqu'il le pousse à produire lui-même une série de représentations, de souvenirs ou d'imagesémotions, repose notamment sur ce que Kluge appelle la « Mischform ${ }^{46}$ », la forme de mélange qui confronte le public à des séquences documentaires tout autant qu'à des mises en scène très théâtrales. Rappelant par ces mélanges que « les contes et la sociologie ne sont pas incompatibles ${ }^{47}$ ", le réalisateur espère amener le spectateur à protester par la production d'émotions contre le froid du réel brut. Il ne faut toutefois pas s'y tromper. Tout comme c'est le cas pour sa théorie des émotions, il ne s'agit pas d'un mouvement dialectique entre deux pôles opposés qui permettraient de distinguer - et de choisir une fois pour toutes - entre documentaire et fiction. Pas plus que le seul rêve ne suffit à fuir une fois pour toutes le réel dur et cruel, car il servirait alors de masque à ce que Kluge nomme «l'exportation de problèmes ${ }^{48}$ » (« Problemexport»), à savoir une pure fuite. C'est en réalité à une interdépendance non dialectique du discours documentaire et de la mise en scène fictionnelle que le réalisateur songe.

Au choc de l'IVG filmée dans Travaux occasionnels d'une esclave (1973), Kluge répond par l'histoire de Gaspard, sauvé in extremis (des eaux) par l'intervention providentielle d'un oiseau qui le ramène sain et sauf dans sa famille. Contre « l'hyperdocumentarité » de la séquence de l'avortement, qui devrait laisser le spectateur las et déçu, Kluge propose "l'hyper-théâtralité » d'un conte raconté en rimes et en ombres chinoises. L'ouverture de Die Macht der Gefühle (1983) nous confronte à une rencontre analogue: après les plans rapprochés et contemporains d'un enfant grièvement brûlé, le spectateur découvre un extrait des Nibelungen de Fritz Lang (1924), montrant également un enfant dans la tourmente, mais selon une tout autre tonalité cette fois. Cette interdépendance entre le désespoir et l'espoir (c'est-à-dire entre le désespoir et la protestation) trouve aussi à s'exprimer, dans Die Macht der Gefühle, dans l'interview d'un chanteur d'opéra qui ne sait pas encore qu'il va mourir inévitablement au cinquième acte (voir plus haut ${ }^{49}$ ), un espoir implicite que semblent confirmer les nombreuses scènes de répétition et de « résurrection » dans le film.

p. 7; Michael Dost, Florian Hopf, Alexander Kluge, « Lebendige Phantasiearbeit und tote Organisation », in Eder, Kluge, Ulmer Dramaturgien, p. 49; Alexander Kluge, « Der Dokumentarfilm als Gefangener der Auftragsproduktion », in Alexander Kluge (éd.), Bestandsaufnahme: Utopie Film. Zwanzig Jahre neuer deutscher Film, Frankfurt am Main, Zweitausendeins, 1983, p. 161-166.

${ }^{46}$ Kluge, « Ein Hauptansatz », p. 7.

47 « Ähnlich ergänzen einander, richtig angewendet, die musikalisch-poetisch erzählenden und die dokumentierenden Formen des Films. Auch hier ist es die Mischform, die zwischen Dokument und Fiktion, zwischen Montage und ungekürzter Wiedergabe, zwischen Phantasie und Wirklichkeitssinn vermittelt. Soziologie und Märchen sind eben nicht, wie man annimmt, Gegensätze, sie sind Pole in ein und derselben Sache, die verschieden aussieht, je nachdem, ob man von der Fähigkeit des Menschen, es mit den Fakten auszuhalten, oder von seiner Fähigkeit, Wünsche zu bilden, ausgeht. » (Ibid.).

48 Günther Hörmann, Maximiliane Mainka, Alexander Kluge, « Politik, Philosophie, Film », in Eder, Kluge, Ulmer Dramaturgien, p. 50.

${ }^{49}$ Récemment encore, dans l'interview fictive de Carmen in Speichersdorf (2013), Kluge a imaginé une directrice d'opéra qui rejoue inlassablement Carmen parce qu'il se pourrait que l'irruption d'un ours polaire dans l'arène ou qu'une pluie diluvienne offre soudain une autre issue à l'opéra qui, selon les mots de Kluge, fait mourir Carmen sous la dague de Don José une fois par jour, quelque part dans le monde. 
À la fois réalisations (par la rencontre de matériaux hétérogènes aux rimes visuelles ou thématiques évidentes) et illustrations du principe cinéma, les séquences rapidement évoquées ici permettent de résumer l'efficacité politique du cinéma selon Kluge: par sa capacité à imiter la production d'images-émotions dans l'esprit du spectateur, le cinéma réactive la « confiance originelle », c'est-à-dire la capacité protestataire, originelle et inaliénable, de tout sujet. Mais le dire de la sorte, c'est s'exposer à ce que Thomas Elsaesser appelait la « ventriloquie » qui guette tout analyste et lecteur de Kluge, qui en viendrait à expliquer et à paraphraser sa singulière théorie des émotions en vase $\operatorname{clos}^{50}$. Pour en sortir tout en mettant cette pensée à l'épreuve de son propre bénéfice, il faut donc se demander, plus avant, comment l'adjonction d'images hétérogènes pourrait suffire à préserver le spectateur de la passivité provoquée par le choc de l'image documentaire difficile ou de l'évasion stérile dans la pure fiction. Et plus largement: comment le cinéma, et singulièrement le cinéma d'un auteur qui truffe littéralement ses films de références savantes, populaires et d'autoréférences qui touchent a priori seulement l'œil de l'initié, peut-il entraîner le spectateur à raviver la production d'émotions ${ }^{51}$ ?

Poser le problème en ces termes revient à s'interroger sur les conditions dans lesquelles le sens unique réalisateur-spectateur est rompu et peut effectivement déboucher sur un travail collaboratif de production des émotions qui dépasse le seul rêve ou le seul choc traumatique imposé par Kluge à son public ${ }^{52}$. Ou encore: comment l'auteur et les spectateurs peuvent-ils collaborer alors que, de toute évidence, ils ne parlent pas le même langage?

${ }^{50}$ Thomas Elsaesser, «A Thousand Coincidences that Afterwards We Call Fate. On Belatedness in Alexander Kluge », conférence prononcée dans le cadre du colloque Reading/Viewing Alexander Kluge's Work, Université de Liège, 11 décembre 2013. Reproduite en traduction française dans ce volume sous le titre « Cent mille hasards qu'après coup on appelle destin ».

${ }^{51}$ C'est, à un autre niveau, la question que posait déjà en 1983 Burghard Schlicht dans sa critique de Die Macht der Gefühle parue, à la sortie du film, dans le Spiegel: « Warum sind Alexander Kluges Filme so schwer zu verstehen? [...] Kluge macht den Vorschlag, der Zuschauer solle sich aus dem überreichen Angebot das aussuchen, was bei ihm bestimmte Assoziationsketten auslöse. [...] Ich bezweifle, daß das über das übliche Maß hinaus (wie der Zuschauer das bei jedem Film macht) möglich ist. Zudem bietet "Die Macht der Gefühle", anders als die "Tagesschau”, wenn man einmal diesen rohen Vergleich anstellt, einen objektiven Sinnzusammenhang, den man, wie ich, ausgerüstet mit Textbuch, Gedächtnisprotokoll und Kluges Schriften, rekonstruieren kann. Aber wer macht sich schon diese Arbeit? [...] Das erschwert die von Kluge gewünschte "Offenheit" seiner Filme, den Zugang zum Publikum, und ist offensichtlich doch unvermeidlich: "Fehler", die man machen muß. Vermeidbar aber erscheint mir die Verwendung einer bestimmten Begrifflichkeit, die das gefühlsmäßige Verstehen - und Kluge selbst versteht das Kino als ein Medium der Gefühle - verhindert. » (Burghard Schlicht, « Gefühle glauben an einen glücklichen Ausgang », Der Spiegel, n³9, 1983, p. 226-231).

52 Kluge s'est lui-même emparé de cette question à plusieurs reprises, en demandant notamment que son vis-à-vis lui fasse confiance, qu'il s'arrête un instant pour prendre le temps de regarder le film, qu'il lui accorde provisoirement le bénéfice du doute, qu'il lui fasse confiance le temps du film, en admettant précisément qu'il pourrait faire fausse route et commettre des erreurs. Voir notamment: Alf Brustellin, Rainer Werner Fassbinder, Alexander Kluge, Volker Schlöndorff, Bernhard Sinkel, « Deutschland im Herbst: Worin liegt die Parteilichkeit des Films? », in Ästhetik und Kommunikation, 32, n 6, juin 1978, p. 124 . 


\section{L'efficacité du cinéma des émotions : le «principe Ferenczi » dans Die Macht der Gefühle}

Dans Die Macht der Gefühle, une longue section, qui couvre grosso modo la première moitié du film, est une citation appuyée de Ferenczi. Ce passage s'ouvre par une intervention de la voix off (de Kluge) : « Les gens peuvent endurer longtemps une chose qu'ils ne supportent plus. Puis soudain ils explosent de façon inattendue et brutale. » Kluge enchaîne avec la première scène de procès du film, où une femme est jugée pour avoir tiré sur son mari alcoolique, qui l'a délaissée pour entretenir une relation amoureuse avec leur propre fille. Bien entendu, cette scène de tribunal est une autocitation: elle rappelle la scène, évoquée au début de ce texte, où Anita Grün est jugée pour le vol d'un pull en plein été. On trouve dans les deux scènes la même incompréhension, le même dialogue de sourds entre le juge et la protagoniste du film (le juge : « on tourne en rond »). Le juge est pourtant de bonne volonté, pourrait-on dire. On a l'impression qu'il s'est fait un tour de rein en essayant de comprendre ce qu'il n'hésite pas à appeler la « logique » du coup de feu de l'accusée. Et même pendant l'audience, il se plie dans tous les sens pour comprendre comment celle-ci a réussi à tourner l'arme contre elle avant de la braquer en direction de son mari. Rien n'y fait: le juge n'arrive pas à expliquer l'acte - le moment de l'explosion autrement que comme une suite d'actes volontaires. Mais, en même temps, si on s'intéresse à la cause du procès, cette scène est une citation de Ferenczi et d'un de ses travaux les plus célèbres, «Confusion de langue entre les adultes et l'enfant ${ }^{53}$ ». Dans la séquence consacrée à la destruction de Babylone ${ }^{54}$, en conclusion de la section du film qui nous intéresse ici, Kluge cite littéralement le titre de Ferenczi, après que le terme de «confusion » a déjà été prononcé à plusieurs reprises dans l'interrogatoire du juge ${ }^{55}$. Pour le dire vite, dans sa conférence de 1932 (publiée

${ }^{53}$ Sandor Ferenczi, « Confusion de langue entre les adultes et l'enfant. Le langage de la tendresse et de la passion» [ «Sprachverwirrung zwischen den Erwachsenen und dem Kind. Die Sprache der Zärtlichkeit und der Leidenschaft »] [1932], in Psychanalyse IV. Euvres complètes, 1927-1933, Paris, Payot, 1982, p. 125-135.

${ }^{54}$ Dans la Bible, Babel signifie précisément «mélange, confusion des langues ». Kluge ne fait ainsi qu'appuyer l'allusion de Ferenczi au célèbre récit biblique. On rappellera que cette traduction « hébraïque » est fautive. En mésopotamien, Babel signifiait originellement « la porte des dieux ». On rappellera également que l'interprétation de cet épisode du récit des origines de l'humanité peut donner lieu à deux lectures opposées: celle qui, dénonçant la confusion des langues, voit dans la présomption humaine le signe du péché; celle qui, au contraire et paradoxalement, voit dans le «malentendu babélien » (et dans la diversité des langues qui en résulte) un rapprochement des hommes, dans leur état de déchéance et de séparation, dans une sorte d'histoire de la communication. Sur cette question, que nous ne pouvons présenter ici que schématiquement, voir, notamment, l'article de James Dauphiné, « Le mythe de Babel », Littératures plurielles, ${ }^{\circ} 1$ 1, 1996, p. 163-173. Pour une interprétation récente de Babel chez Kluge, menée à partir de Kafka, cf. Alexander Kluge, «"Un désir d’issues”: onze réponses au sujet de Kafka (Entretien), in Jean-Pierre Morel, Wolfgang Asholt (dir.), Franz Kafka, Paris, L'Herne (Cahiers de l'Herne, 108), 2014, p. 21-22.

${ }_{55}$ Envisagée de cette façon, cette séquence de tribunal de Die Macht der Gefühle est absolument fondamentale. Comme dans d'autres films de Kluge, on y voit à l'œuvre une mise en question du dispositif juridique. En effet, le malentendu entre le juge et l'accusée vient du fait que de telles scènes de confusion entre tendresse enfantine et passions adultes sont précisément l'occasion pour l'enfant, par identification, d'intérioriser le sentiment de culpabilité de l'adulte (voir Ferenczi, «Confusion de langue », p. 130). Ce 
en 1933), Ferenczi reprend à son compte la théorie de la séduction par laquelle le jeune Freud avait d'abord essayé, entre 1895 et 1897, d'expliquer les névroses de ses patientes. Il ne peut être question ici de s'appesantir sur le destin de cette théorie dans l'œuvre de Freud lui-même. On en retiendra, en premier lieu, que cette théorie de la séduction, dont Die Macht der Gefühle met en scène un cas exemplaire, décrit une situation « où le sujet (généralement un enfant) subit passivement, de la part d'un autre (le plus souvent un adulte), des avances ou des manœuvres sexuelles ${ }^{56} \gg$. Il s'agit, selon Freud lui-même, d'une « expérience purement passive ${ }^{57}$ » où le sujet, « encore incapable d'émotion sexuelle », subit la scène parce qu'elle ne peut pas " évoquer chez lui de réponse ${ }^{58}$ ». Ce que le sujet vit est vécu sans préparation, dans la mesure où aucune représentation d'ordre sexuel préalable n'existe pour rendre cet événement compréhensible. Pour le dire de façon un peu plus métaphorique, l'enfant ne connaît pas la langue de l'adulte qui fait véritablement « effraction ${ }^{59}$ » dans son expérience.

Il ne faudrait cependant pas limiter la référence que Kluge fait à Ferenczi à une approche thématique. Avant 1983, Kluge avait déjà cité au moins une fois Ferenczi, en 1970, dans le cadre d'une émission sur la troisième chaîne allemande, consacrée au rapport entre " Cinéma et société », une émission restée célèbre parce qu'elle fut interrompue après quarante-cinq minutes par l'intervention directe sur le plateau du rédacteur en chef accusant Kluge de "manipuler l'émission ${ }^{60}$ ». L'émission avait pris un tour particulier: le débat avait glissé de la question de l'efficacité sociale du cinéma vers une mise en question du formatage des émissions de télévision qui prétendent s'adresser au grand public et le rendre plus intelligent. À rebours, le rédacteur de l'émission reproche à Kluge de faire des films remplis de citations qui sont inaccessibles à la plupart des spectateurs. Le débat est impossible, mais la

qui n'était qu'un jeu du point de vue de l'enfant devient le motif d'une punition, en même temps que l'enfant, confronté à l'hypocrisie de l'adulte auquel il s'est identifié, se retrouve « clivé, à la fois innocent et coupable » (ibid., p. 131). Il n'est donc pas étonnant que le juge insiste sur la confusion dans laquelle se trouve l'accusée et que celle-ci ne puisse se déclarer ni coupable ni innocente.

${ }^{56}$ Jean Laplanche, Jean-Bernard Pontalis, «Séduction (scène de -, théorie de la -) », in Vocabulaire de la psychanalyse, p. 436.

57 Cf. Sigmund Freud, La naissance de la psychanalyse: lettres à Wilhelm Fliess: notes et plans (1887-1902), Paris, PUF, 1956.

58 Laplanche, Pontalis, « Séduction (scène de -, théorie de la -) », p. 436-437.

59 Laplanche et Pontalis font référence à Ferenczi dans leur notice p. 439.

${ }^{60}$ L'enregistrement de cette émission a été édité en DVD sous le titre Reformzirkus. Le texte du débat est reproduit intégralement sur le site: http://fr.scribd.com/doc/99448152/Reformzirkus-1970. Kluge fait référence à Ferenczi, précisément à l'article sur la confusion des langues dans l'extrait suivant: "Halt mal! Die Gedanken sind kompliziert. Ich beziehe mich jetzt darauf, dass die libidinöse Besetzung, die Menschen haben, und zwar seit der Höhlenmalerei oder seit der ersten Liebesbeziehung, die sie überhaupt haben, dass diese, gewiss auch zugerichtete und deformierte Libido, dass die nach Ausdruck verlangt. Alles, was diesen Ausdruck herstellt, hat gleichzeitig mit Leben zu tun und könnte gleichzeitig subsumiert werden unter Kunst. Es ist nur so, dass die Freiheit hierfür nur in den herrschenden Schichten war. Aber das besagt nicht, dass das Grundbedürfnis, das Interesse an Ausdruck, nicht überall vorhanden ist. Sprache - wenn eine Mutter mit ihrem Kind spricht - und Ferenczi hat ja über die Missverständnisse da ein Buch extra geschrieben, über die Sprachfähigkeit von Kindern und von Müttern in der frühkindlichen Sozialisationsphase. DIESE FORMEN SIND ALLE IN DIESEM SINNE KUNST. DA JA KUNST DEFORMIERT IST... ». 
caméra continue de tourner malgré l'agitation régnant sur le plateau et malgré la convocation de tous les techniciens et rédacteurs à venir constituer le public auquel le WDR est censé s'adresser.

Dans ce contexte, la citation improvisée - on est après la première interruption du programme ${ }^{61}$ - mais essentielle de Ferenczi prend une signification précise. En 1970, Kluge revient en effet au cinéma quelques mois après la mort d'Adorno. Pendant plusieurs années, il s'était consacré à d'autres projets, menés collectivement, comme la création d'un jardin d'enfants et l'enregistrement direct de la langue des classes populaires et des ouvriers. Mais, peut-être précisément parce qu'Adorno vient de mourir, il ne peut alors être question pour Kluge de renoncer au projet, philosophique et sociologique, de la Théorie critique. C'est d'elle qu'il faut partir, ne cesse de répéter Kluge, si on veut inventer les métaphores d'une pensée qui affronte sa bêtise et qui cherche à «s'articuler », c'est-à-dire qui se donne le temps de la réflexion et s'autorise à formuler une idée complexe.

C'est certainement sur ce point que la référence à Ferenczi est déterminante. On ne peut en effet manquer de se rappeler, à ce stade, les critiques sévères qu'Adorno avait adressées depuis le milieu des années 1950 à une psychanalyse d'inspiration ferenczienne. Dans Dialectique négative, en 1966, Adorno reproche à Ferenczi d'avoir concrètement restreint la « critique implacable du surmoi » qui constituait pour lui, dans son «époque héroïque ${ }^{62}$ », l'apport fondamental de la psychanalyse. Pour Adorno, en effet, seule une critique radicale du surmoi, sa déconstruction, permet à la psychanalyse de mener à bien la critique de la société qui produit ce surmoi. À défaut, la psychanalyse ne fait que reconnaître et avaliser « l'intériorisation aveugle et inconsciente de la contrainte sociale ${ }^{63} »$. Autrement dit, dans ce cas, la psychanalyse «consent à la norme sociale dominante » quand elle ne se met pas tout simplement au service d'une « adaptation inconditionnelle ${ }^{64} »$ des individus à la société. En son fond, cette critique de Ferenczi par Adorno résume la critique que le philosophe adressait une décennie plus tôt aux développements récents de la psychanalyse (et des sciences sociales) aux États-Unis vers des perspectives pédagogiques et éducatives adaptatives et normalisantes ${ }^{65}$.

Adorno resserre cette critique dans son article important, écrit pour un volume d'hommage offert à Horkheimer, intitulé « À propos du rapport entre sociologie et psychologie ${ }^{66} »$. Les cibles désignées de l'article sont Talcott Parsons, cité d'emblée, Karen Horney et Erich Fromm, ainsi qu'Anna Freud ${ }^{67}$. Mais ces cibles circonstancielles

${ }^{61}$ Avant cette interruption, Kluge avait évoqué en début d'émission Hegel, Marx et, à plusieurs reprises, Adorno.

62 Theodor W. Adorno, Dialectique négative, trad. de l'all. par le Groupe de traduction du Collège de philosophie: Gérard Coffin, Joëlle Masson, Olivier Masson et al., Paris, Payot, 2001 [1966], p. 329.

63 Ibid.

64 Ibid., p. 131.

${ }^{65}$ Theodor W. Adorno, La psychanalyse révisée, trad. de l'all. par Jacques Le Rider, Paris, Éditions de l'Olivier, 2007 [1946, 1952].

66 Theodor W. Adorno, "À propos du rapport entre sociologie et psychologie », in Société: Intégration, Désintégration, textes trad. de l'all. et de l'angl. par Pierre Arnoux, Julia Christ, Georges Felten et al., Paris, Payot, 2011, p. 315-367.

${ }^{67}$ À ce niveau, les critiques d'Adorno rejoignent celles de Marcuse à la même époque. Il s'agit 
ne peuvent cacher les véritables destinataires de la critique qu'Adorno formule contre les usages de la psychanalyse par la sociologie, et en particulier par des sociologies qui se veulent des théories critiques de la société. Ainsi, à trente ans de distance ${ }^{68}$, Adorno ne ménage pas ses critiques à l'égard d'un Marcel Mauss et de son projet de réaliser une anthropologie de l' « homme complet » ou de l' « homme total » dans une société qui est elle-même « totalitaire ${ }^{69} »$. L'argument est polémique et certainement discutable tant en ce qui concerne les intentions de Mauss qu'en ce qui a trait au potentiel critique de sa sociologie et des usages qui en ont été faits. Il faut toutefois en retenir le soupçon qu'Adorno fait peser sur des pratiques d'alignement les uns sur les autres des différents savoirs humains. Dans le même élan, Adorno en vient à formuler une critique du même ordre à l'égard de la psychanalyse de Ferenczi. Le passage suivant associe Mauss et Ferenczi dans le même reproche:

Der integrale Mensch, der die private Divergenz der psychologischen Instanzen und die Unversöhnlichkeit der Desiderate von Ich und Es nicht mehr spürte, hätte damit die gesellschaftliche Divergenz nicht in sich aufgehoben. [...] Seine Integration wäre die falsche Versöhnung mit der unversöhnten Welt, und sie liefe vermutlich auf die «Identifikation mit dem Angreifer » hinaus, bloße Charaktermaske der Unterwerfung. ${ }^{70}$

Adorno présente certes Ferenczi comme «peut-être le plus imperturbable et le plus libre de tous les psychanalystes ${ }^{71} »$. Mais c'est en réalité pour faire porter le soupçon sur la psychanalyse depuis ses débuts. Adorno n'hésite pas à reprendre la critique formulée par les révisionnistes à propos de la « froideur analytique ${ }^{72}$ » afin de porter le fer contre la pente originairement pédagogique de la psychanalyse. Or, il ne pouvait pas ignorer que Ferenczi avait signé son entrée en psychanalyse par un article sur «Psychanalyse et pédagogie». Ferenczi y menait alors une critique radicale de toute forme d'éducation - autrement dit de «négation » des émotions ${ }^{73}$. Adorno considère qu'ensuite Ferenczi aurait reculé.

Dans Die Macht der Gefühle, Kluge s'appuie pour sa part sur les derniers textes de Ferenczi afin de montrer, au contraire, que le psychanalyste hongrois a

pour les deux philosophes de faire un « retour à Freud», notamment aux considérations freudiennes sur la pulsion de mort, contre ce qu'ils considèrent comme les tendances révisionnistes de la psychanalyse américaine. Voir Jean-Marc Durand-Gasselin, L'École de Francfort, Paris, Gallimard, 2012, p. 209-221. Voir aussi Paul-Laurent Assoun, L'École de Francfort, Paris, PUF, 2012 [1987], p. 103-107.

${ }^{68}$ Mauss avait publié en 1924 un article sur les « Rapports réels et pratiques de la psychologie et de la sociologie », in Sociologie et Anthropologie, p. 281-310. Sur le rapport de radicalisation critique d'Adorno envers Mauss, voir Grégory Cormann, Jeremy Hamers, « Kluge, Adorno et l'indomptable Leni Peickert », Cahiers du GRM [en ligne], n 5, 2014, http://grm.revues.org/412.

69 « Jedes Menschenbild ist Ideologie außer dem negativen. Wird heute etwa gegenüber den mit der Arbeitsteilung verfilzten Zügen der Spezialisierung an den Vollmenschen appelliert, so verspricht man dem Undifferenzierteren, Gröberen, Primitiveren eine Prämie und verherrlicht am Ende die Extroversion des go-getters, jene, die abscheulich genug sind, um im abscheulichen Leben ihren Mann zu stehen ». Theodor W. Adorno, «Zum Verhältnis von Soziologie und Psychologie» [1955], in Soziologische Schriften, vol. I, Darmstadt, Wissenschaftliche Buchgesellschaft, 1998, p. 67; pour la traduction française, cf. Adorno, « À propos du rapport », p. 342.

70 Adorno, «Zum Verhältnis », p. 65-66; « À propos du rapport », p. 340.

71 Adorno, « À propos du rapport », p. 339.

72 Ibid., p. 361.

73 Sandor Ferenczi, « Psychanalyse et pédagogie » [1908], in L'enfant dans l'adulte, p. 29-39. 
maintenu jusqu'au bout sa radicalité, en acceptant de la retourner, à la charnière des années 1920-1930, contre sa propre pratique de la cure analytique ${ }^{74}$. Revenons par conséquent à l'article de Ferenczi sur « La confusion des langues ». Dans ce texte, Ferenczi retourne le miroir vers le psychanalyste et vers le rapport qu'il instaure dans la cure avec son patient. La vigilance du psychanalyste doit être double: il doit certes identifier les résistances que le patient met en œuvre pour éviter de regarder son passé en face; mais il doit, en même temps, et de façon aussi importante, « deviner» les critiques que le patient aurait à lui adresser sans pouvoir le faire, sans que le cadre de la cure lui permette d'exprimer ses critiques (les patients s'identifient au psychanalyste, ils ne veulent pas le décevoir ou lui déplaire, etc.). La phrase citée plus haut, selon laquelle « on peut endurer longtemps ce qui nous est insupportable », prend alors un autre sens: elle ne concerne plus le traumatisme initial, mais la situation même de la cure. À ne pas y être attentif, le psychanalyste s'expose dans ses séances à ne faire que « répéter le trauma », qu'à provoquer chez son patient une crise d'angoisse extrême, une « explosion de colère et de fureur ${ }^{75}$ » qui met son patient dans un état presque inconscient. La sentence autocritique est cinglante: la réserve du psychanalyste, son « attitude froide et pédagogique ${ }^{76}$ » face à ce type d'explosion brise le lien avec le patient.

La solution envisagée par Ferenczi consiste du coup à renverser la situation analytique: à savoir reconnaître la supériorité du patient, qui perçoit de façon très fine les souhaits et les humeurs du psychanalyste; il s'agit d'admettre ses propres erreurs $^{77}$, d'y renoncer et de permettre au patient de formuler ses critiques. Dans ces conditions, le psychanalyste restaure une relation de confiance avec son patient sur un plan qui n'est plus théorique mais bien émotionnel. Dans un autre article essentiel, Ferenczi parle du «tact» et de $l^{\prime}$ «empathie ${ }^{78} »$ du psychanalyste. C'est certes «à l'aide de [son] savoir » que le psychanalyste peut identifier les «associations » que le patient ne perçoit pas encore ${ }^{79}$. Il faut cependant du tact pour ne pas « stimuler la résistance du patient inutilement ou intempestivement ${ }^{80}$ " et l'amener progressivement à renoncer aux mécanismes de défense qui l'ont

${ }^{74}$ Notons qu'A. Kluge recourt à son tour au concept d'identification ou d'« introjection de l'agresseur » dans son film de 2008 Nachrichten aus der ideologischen Antike, où il reprend le projet d'Eisenstein de porter Le Capital de Marx au cinéma. Voir Alexander Kluge, Idéologies: des nouvelles de l'Antiquité. Marx - Eisenstein - Le Capital, trad. de l'all. par B. Vilgrain, Courbevoie, Théâtre Typographique, 2014, p. 16.

${ }^{75}$ Ferenczi, « Confusion de langue », p. 126-127.

${ }^{76}$ Ibid., p. 129.

77 Ferenczi se réjouit que le psychanalyste «commette de toute façon suffisamment d'erreurs » (ibid., p. 128) afin de détraquer une cure qui risquait de «trop bien réussi[r]» (ibid., p. 126).

78 Sandor Ferenczi, « Élasticité de la technique psychanalytique » (1928), in Psychanalyse IV, p. 55.

${ }^{79}$ Le psychanalyste est capable de faire ces associations, d'une part, du fait qu'il a "disséqué » de nombreux psychismes humains, mais surtout parce qu'il s'est soumis, dans le cadre de la cure « didactique », à la « dissection de son propre Moi ». Toutefois, cette analyse didactique, qui a aussi eu pour conséquence rapide de standardiser la pratique analytique, doit, on le voit, s'appuyer structurellement sur ce que Ferenczi appelle le « reste pas encore résolu de l'équation personnelle » du psychanalyste, c'est-à-dire sur les « erreurs » qu'il a commises et continue de commettre.

${ }^{80}$ Ibid. 
empêché jusque-là de «s'adapter à une réalité pleine de déplaisir ${ }^{81}$ ». La phrase déjà deux fois commentée de Die Macht der Gefühle (« on peut endurer longtemps une situation insupportable ») prend ainsi une troisième signification: le résultat de la psychanalyse selon Ferenczi est d'《 apprendre à supporter une souffrance ${ }^{82}$ ». Ce faisant, Ferenczi pense n'avoir rien fait d'autre que retrouver le principe même de la psychanalyse qui s'en remet aux associations libres produites par le patient. En réalité, souligne-t-il, ce principe est double: d'une part, il « oblige le patient à s'avouer des vérités désagréables », mais, d'autre part, il «l'autorise à une liberté dans la parole et dans l'expression des sentiments ${ }^{83} »$.

La question de l'antidote à la passivité du spectateur par laquelle nous clôturions provisoirement notre recherche de l'efficacité politique d'un cinéma des émotions peut à présent être reformulée de façon plus précise: comment, dans Die Macht der Gefühle, la remise en jeu de Ferenczi a-t-elle permis à Kluge de libérer son spectateur par la production d'émotions, malgré la confrontation à des vérités presque insupportables imposées à ce spectateur, et ce, via un langage qui, a priori, forçait le public à la passivité ? Sans rabattre la réflexion de Ferenczi relative à la relation entre le patient et l'analyste sur la relation entre un réalisateur et ses spectateurs, on dira encore: dans quelles conditions Kluge, non plus malgré son érudition mais « avec » celle-ci, peut-il susciter la production d'émotions croisées chez tout spectateur? Comment peut-il restaurer une relation de confiance avec son spectateur sur un plan qui n'est plus théorique mais émotionnel?

\section{Ouverture}

En guise de première réponse à cette question, nous voudrions formuler l'hypothèse suivante: par l'emploi de la Mischform, par la convocation bigarrée de différents types et registres de représentations, Kluge tente de passer outre à l'inégalité (des compétences) entre l'auteur et le public pour tendre à une production déhiérarchisée de sentiments. Ses déclarations d'amour répétées pour une période de l'histoire du cinéma qu'il n'a pas connue donnent quelque consistance à cette proposition. Kluge voue un amour particulier à l'époque de la diversité primitive, un premier âge cinématographique dans lequel, dit-il, le septième art était fait de « hasard, de caractère sérieux [...], de génie, d'incompétence et de chance ${ }^{84} »$. À cette époque, « [o]n essayait sans cesse d'inventer le cinéma. Et chaque fois qu'il était inventé, on l'inventait encore une fois ${ }^{85} »$. Dans le cinéma qu'il met progressivement en place jusqu'au début des années quatre-vingt - et dont Die Macht der Gefühle est peut-être un aboutissement -, Kluge essaye de rejouer cette diversité primitive. Non pas par

81 Sandor Ferenczi, « Principe de relaxation et néocatharsis » [1930], in Psychanalyse IV, p. 89.

${ }^{82}$ Ferenczi, « Élasticité de la technique psychanalytique », p. 55.

83 Ferenczi, « Principe de relaxation et néocatharsis », p. 89.

${ }^{84}$ Kluge, Geschichten, p. 7.

85 « Das war ein pausenloser Versuch, den Film zu erfinden, und immer, wenn er erfunden wurde, wurde er noch mal erfunden." («Alexander Kluge», in Edgar Reitz, Bilder in Bewegung. Essays. Gespräche zum Kino, Reinbek bei Hamburg, Rowohlt Taschenbuch Verlag, 1995, p. 82). 
nostalgie d'un cinéma précédant les «sens uniques commerciaux ${ }^{86}$ », mais par amour d'une situation dans laquelle tout le monde, réalisateurs comme spectateurs, était logé à la même enseigne. Un dialogue de sourds généralisé en somme, en vertu duquel la liberté, l'erreur et la production d'émotions étaient partagées par les spectateurs et les réalisateurs (producteurs, opérateurs, acteurs, etc.) qui créaient des œuvres historiquement imprévisibles pour un public tout aussi imprévisible. Une relation émotionnelle de pleine confiance, inévitablement non hiérarchisée parce qu'incapable de recourir à des savoirs, mécanismes et sens uniques préétablis.

La tension identifiée au départ de notre réflexion entre la conception adornienne d'un sujet ayant totalement incorporé la froideur, et celle, défendue par Alexander Kluge, d'un être humain pétri d'une résistance émotionnelle au froid, reste irrésolue. Deux propositions conclusives peuvent toutefois être tirées de ce qui précède. La première lie la diversité primitive à l'espoir défendu par le dernier Adorno dans son texte « Résignation ${ }^{87}$ ». Dans ce court article lu d'abord à la radio quelques mois avant sa mort, Adorno lie l'exil ou le retrait élitiste, qu'il semblait avoir défendu tout au long de sa vie, à une forme d'espoir pour les générations futures. Affirmant que « [c]e qui a été pensé de manière prégnante doit être pensé ailleurs, par d'autres : cette confiance accompagne même la pensée la plus solitaire et la plus impuissante ${ }^{88}$ », le philosophe francfortois justifie son refus d'une fonctionnalisation de sa pensée (par le mouvement étudiant en l'occurrence), en faisant appel à un futur dans lequel quelqu'un énoncera une pensée proche de celle de l'intellectuel retiré. En rejouant la diversité primitive dans son cinéma des associations, Kluge réalise cet espoir; il lui offre une concrétisation en le déplaçant dans le domaine du cinéma. Autrement dit, il est de ceux qui, dans son futur, " proviennent d'Adorno ».

La seconde proposition conclusive que nous voudrions esquisser ici suggère que c'est bien un « rapport à la réalité » qu'Adorno a voulu préserver en se retirant, paradoxalement, d'un monde qui risquait de l'en priver par excès de froideur. En première lecture, cette posture permet au penseur de tourner le dos au réel pour ne pas voir sa pensée et sa sensibilité réduites à leur mise en action ${ }^{89}$. Et il serait sans doute tentant de reconnaître ici un retrait quasi enfantin ${ }^{90}$. En réalité pourtant, ce retrait rejoue aussi l'interdépendance étroite identifiée par Ferenczi entre souvenir de toute-puissance et sens de réalité contemporain de la naissance. Car, se retirer - avec l'espoir qu'un jour quelqu'un d'autre, ailleurs, pensera à nouveau - c'est aussi

${ }^{86}$ Ibid.

87 « Was triftig gedacht wurde, muß woanders, von anderen gedacht werden: dies Vertrauen begleitet noch den einsamsten und ohnmächtigsten Gedanken » (Theodor W. Adorno, " Resignation », in Kritik. Kleine Schriften zur Gesellschaft, Frankfurt am Main, Suhrkamp, 1971, p. 150). Pour une traduction française du texte, voir: Theodor W. Adorno, « Résignation », trad. de l'all. par N. Gabriel, A. Birnbaum, M. Métayer, Tumultes, $\mathrm{n}^{\circ}$ 17-18, 2002, p. 173-178.

88 Ibid., p. 178.

${ }^{89}$ Cette inquiétude d'Adorno est également manifeste dans « Éduquer après Auschwitz », p. 239-240.

${ }^{90}$ Cette lecture du retrait et d'un « rejeu » de la toute-puissance de l'enfant entretient quelque analogie avec 1'hypothèse défendue par Ernst Bloch - dont Kluge se distancie explicitement - d'une évasion maîtrisée que l'enfant produit quotidiennement dans les jeux de ses premières années (« S'évader tous les jours »). Ernst Bloch, Le principe espérance, trad. de l'all. par F. Wuilmart, Paris, Gallimard, 1976, p. 34-35; sur le positionnement de Kluge par rapport à Bloch et Adorno, cf. Kluge, Rack, « Erzählen ». 
conserver, de facto, une pensée qui pourra encore, un jour, agir sur le monde. Comme chez Ferenczi, la résistance de l'enfant est une réponse donnée au monde réel et non la tentative de rejouer une situation perdue à jamais; le retrait d'Adorno, son refus des mots d'ordre et d'une mise en action des idées, visent avant tout la conservation d'une pensée capable d'exister un jour dans le réel. C'est en substance ce que Kluge explique lorsqu'il insiste, confronté au paradoxe d'un Adorno d'apparence froide et pourtant pétri de confiance originelle ${ }^{91}$, sur l'origine de son espoir, nécessairement contemporaine d'un retrait:

Er spricht vom Antirealismus des Gefühls. Keiner hält unerträgliche Verhältnisse für geeignet in ihnen zu leben. Ich habe ein Eigentum an meinen Gefühlen, an meiner Libido. Und irgendwas draußen will daran Eigentum bilden. Und dagegen wehre ich mich mit allen Kräften. Diese Unruhe ist eigentlich die Unruhe im Werk von Adorno. Die Negation wird getrieben von Sehnsucht und aus kindlicher Kenntnis, dass es eine Alternative gibt. [...] Es kommt aus der Kindheit. Ich kenne keinen anderen Menschen, dem man in den Augen eine solche Menge von Urvertrauen ansieht wie den Augen von Adorno. Der Glaube, dass es zumindest in den ersten drei Tagen seines Lebens gut mit ihm gemeint war, der haftet ihm lebenslänglich an. ${ }^{92}$

${ }^{91}$ Nous retrouvons ici la question de la confiance - une confiance inquiète - qui traversait dès 1944 La Dialectique de la raison. Au terme de ce travail, nous pouvons, d'une part, identifier l'origine de cette confiance dans ce qu'on pourrait appeler le « projet existentiel » d'Adorno et, d'autre part, poser la question (qui dépasse le cadre de cet article) du destin de cette confiance originelle d'Adorno dans la suite de son œuvre, jusqu'à un texte comme « Résignation».

${ }_{92}$ A. Kluge, dans un entretien avec Peter Laudenbach, « Kluge über Adorno », Berliner Tagesspiegel, 11 septembre 2003. L'entretien est consultable en ligne sur le site d'A. Kluge: http://www.klugealexander.de/zur-person/interviews-mit/details/artikel/kluge-ueber-adorno.html. 
